\title{
LA CARTOGRAFÍA ETNOGRÁFICA: UNA METODOLOGÍA PARA EL ESTUDIO DEL POBLAMIENTO DE MISIONES, ARGENTINA
}

\author{
ETHNOGRAPHIC CARTOGRAPHY: \\ AS METHODOLOGY FOR THE STUDY \\ OF MISIONES'S SETTLEMENT, \\ ARGENTINA
}

\section{María Cecilia Gallero}

mariaceciliagallero@gmail.com

Doctora en Historia por la Universidad Nacional de Cuyo

Investigadora Adjunta del Consejo Nacional de Investigaciones Cientificas y Técnicas-CONICETen el Instituto de Estudios Sociales y Humanos (IESyH - CONICET-UNaM)

\section{RESUMEN}

La "cartografía etnográfica" como una metodología innovadora propone complementar la cartografía - por ser una ciencia dedicada al estudio y elaboración de mapas - con la etnografía - por ser una herramienta fundamental para la reconstrucción del territorio como espacio representado y apropiado por los que lo habitan-. Este trabajo se propone mostrar su aplicación a través de un ejemplo concreto, como lo es el poblamiento de la provincia Misiones (Argentina), caracterizada por una diversidad sociocultural inscripta temporalmente en un entramado de relaciones sociales que se distribuyen espacialmente, y que se hizo efectiva a través de la colonización oficial y privada; y espontáneamente según corrientes de migraciones de población de países fronterizos (Brasil y Paraguay). Estas características llevaron a la implementación de una metodología específica que permitiera estudiar dicho proceso de manera apropiada.

Palabras clave: Cartografía. Etnografía. Misiones.

\begin{abstract}
The "ethnographic cartography" as an innovative methodology proposes to complement cartography -for being a science dedicated to the study and elaboration of mapswith ethnography, because it is a fundamental tool for the reconstruction of the territory as a space represented and appropriated by those who inhabit it. This paper aims to show its application through a concrete example, such as the settlement of the Misiones province, Argentina, characterized by a sociocultural diversity temporarily inscribed in a network of social relationships that are spatially distributed, and that became effective through official and private colonization; and spontaneously settlements according to migratory flows of population of border countries -Brazil and Paraguay-. These characteristics led to the implementation of a specific methodology to study the process properly.
\end{abstract}

Keywords: Cartography. Etnography. Misiones. 


\section{INTRODUCCIÓN: CÓMO APORTA EL OBJETO AL MÉTODO}

Un supuesto básico en el oficio del historiador es que «la historia se hace con documentos», los cuales, luego de pasar por la crítica interna y externa, se utilizan como «fuentes» para acceder al pasado y así poder analizarlo y reconstruirlo. Sin embargo, las características propias de los documentos propician que la elección de un método muchas veces incite a pensar algo nuevo para poder abarcar lo que se intenta estudiar.

El problema de la «observación histórica» (BLOCH, 2006: 51 y siguientes) es evidente con relación al «objeto» que se indaga, tal como resultó explorar el proceso de poblamiento de Misiones. Esta provincia constituye un área particularmente relevante para el estudio de los procesos migratorios o de colonización. Un espacio relativamente pequeño $\left(30719 \mathrm{~km}^{2}\right)$, donde habitaban pueblos originarios y luego se asentaron pobladores de más de veinte nacionalidades diferentes, se constituyó en un verdadero «laboratorio» para el estudio de la organización del espacio geográfico, el impacto colonizador, la gestación de la historia regional, los procesos interculturales, la constitución de identidades culturales y las relaciones interétnicas e intraétnicas.

Este trabajo tiene como objetivo analizar y describir la metodología utilizada para estudiar el impacto del proceso de poblamiento sobre la distribución de la tierra en las colonias fundadas por las Compañías Colonizadora Alto Paraná y Compañía Eldorado a través de la reconstrucción de una cartografía histórica y etnográfica desde la creación (1919) hasta la saturación de los lotes coloniales (1969).

Esta metodología comenzó a gestarse cuando el estudio de la inmigración alemana-brasileña, en la Colonia Puerto Rico,trató también de reconstruir su proceso de poblamiento y plasmar la espacialidad de las relaciones sociales,y así poder estudiar la territorialidad como un factor a ser analizado. De modo que la cartografía etnográfica fue una herramienta teórico-metodológica que surgió ante el empeño de plasmar el proceso de poblamiento que gradualmente transformó el territorio. Esta herramienta complementa la cartografía — por ser una ciencia dedicada al estudio y elaboración de mapas - con la etnografía por ser una herramienta fundamental para la reconstrucción del territorio como espacio representado y apropiado por los quienes lo habitan-.

Es de la escuela de los Annales, y en especial de FernandBraudel (BRAUDEL, 2001), que la historia tomó en cuenta la importancia de la larga duración, de la cultura material, del clima, de los métodos agrícolas y de la construcción de los espacios. Implica un enfoque sistémico y transdisciplinario que parte de la geografía histórica (SAUER, 1976; SANTOS, 1996), la antropología social (GUBER, 2004); la etnografía (GUBER, 2014 y 2018) y la historia agraria (BLOCH, 2002 y 2006).

Para poderdesplegar esta metodología, este trabajo desarrolla, a partir de la investigación de campo y de archivo, en una doble perspectiva diacrónica y sincrónica, el impacto del proceso de poblamiento en las relaciones sociales. Por lo tanto, en primer términose realiza un breve estado de la cuestión sobre la cartografía etnográfica, luego se expone cómo se ha puesto en prácticaesta metodología; posteriormente se analiza su aplicación en las tres colonias fundadas por la principal compañía colonizadora de Misiones, para poder concluir con las reflexiones finales. 


\section{ANTECEDENTES DE LA CARTOGRAFÍA ETNOGRÁFICA}

La cartografía etnográfica como metodología es de reciente aplicación, aunqueentre sus antecedentes se pueden mencionar los aportes de Carrera Diez (2007) y Gallero y Kraustofl (2009). Mientras que Carrera Diez señala la cartografía etnográfica como herramienta para observar las complejidades en las interacciones entre «los factores físicos del territorio» $\mathrm{y}$ «el factor humano» en Constantina, Andalucía; Gallero y Kraustofl proponen utilizarla para representar algunos de los procesos de poblamiento e inmigración más importantes para el caso de Misiones entre 1881 y 1970.

En lo que respecta a la «cartografía» y la elocuencia de los mapas, es necesario recordar que estos expresan una manera de concebir, articular y estructurar el mundo humano (HARLEY, 2005: 80) y que se puede hablar de dos categorías de clasificación: el «mapa instrumento», de carácter informativo y práctico; y el «mapa imagen», el cual alberga una abstracción, un esfuerzo intelectual de construcción de un instrumento con fines prácticos, pero revestido también de un carácter intangible como imagen (MONTOYA ARANGO, 2007: 157). En esta última clasificación,se enmarca la cartografía etnográfica, una vez que esta se centra en el mapa como una representación que redescribe el espacio y el territorio.

En el análisis sobre la elaboración cartográfica, se destaca una línea de investigación centrada en el mapa como representación (PICCOLOTTO, 2004) y en el rol de las agencias y de los agentes estatales vinculados a las tareas catastrales y de mensura (GARAVAGLIA y GAUTREAU, 2011).Estosautores señalan la importancia de considerar y problematizar el rol de dichasagencias y agentescuyoavance posibilitó el proceso de producción de un conocimiento específico del territorio bajo el monopolio estatal. En este sentido, esta línea invita a complejizar la mirada sobre la relación entre la capacidad de definir la propiedad de la tierra y los procesos de construcción estatal de un territorio. Línea que ha sido complejizada por Carla LOIS (2014) y que se ha transformado en un punto de partida ineludible para el estudio de la cartografía argentina.

Con relación al proceso de poblamiento de Misiones ${ }^{1}$, un antecedente ineludible es el trabajo de Robert EIDT (1971), quien destaca la condición de frontera o área disponible y la génesis de un poblamiento en el que se describe la influencia de la venta de la tierra realizada por Corrientes en 1881 y las diferencias en los patrones de asentamiento de las colonias. Por su parte, Graciela De Marco realiza una primera aproximación desde la geografía histórica al poblamiento de este territorio (DE MARCO, 1980), y AlfredoBolsicaracteriza desde una perspectiva geográfica-históricael problema de la yerba en su tesis doctoral (BOLSI, 1986), en la cual analiza la influencia de su explotación en el poblamiento de Misiones.

Con relación a la cartografía de Misiones específicamente, Miguel Ángel Stefañuk realizóun profundo estudio relativo a la toponimia, los patrones de asentamiento en la provincia y la influencia de los agrimensores en los procesos de parcelamiento, particularmente tras la federalización del territorio en 1881 (STEFAÑUK, 1995, 1999 y 2009).

Respecto a la «etnografía» sobre los distintos procesos de migración y colonización, es posible destacar una producción académica más abundante para el caso de Misiones. En términos generales, al igual que en la historiografía argentina, en los estudios sobre migración y colonización en esta provincia, se advierte la centralidad del fenómeno migratorio europeo. La fundaciónde la Universidad Nacional de Misiones (UNaM), en 1973, abrió un espacio de 
investigación científico-profesional para el tratamiento histórico, etnográfico y antropológico acerca delos colonos y las colonias en Misiones. La investigación de BARTOLOMÉ (2000) sobre la inmigración polaca y ucraniana procedente de Galitzia (Antiguo Imperio Austro-Húngaro) en la localidad de Apóstoles ha sido un aporte en este sentido. Este trabajo da cuenta de una migración bajo el amparo de la colonización oficial durante la última década del siglo XIX, con base en la cual el autor ha buscado indagar el desarrollo agrario en cuanto a las estrategias adaptativas, variables culturales y el rol de la etnicidad en el comportamiento de estos inmigrantes. Además de los estudios centrados en experiencias particulares de colonización en Misiones, también se encuentran aquellos que han buscado abarcar los procesos de inmigración en el territorio de Misiones de forma más global. Los primeros trabajos de BARTOLOMÉ (1982) y de PERIÉ DE SCHIAVONI Y ZOUVI (1985) han caminado en ese sentido, el primero haciendo hincapié en el desarrollo de la agricultura en el territorio; y el segundo, en las características particulares de los proyectos de colonización estatal y privada que fueron implementados sucesivamente en el territorio, en cuanto a su distribución geográfica y la procedencia de los contingentes de inmigrantes que arribaron en dicho territorio. Procesos de Integración en una Sociedad Multiétnica. La provincia argentina de Misiones (1880-1985), de Roberto ABÍNZANO (1985), es una tesis doctoral aun inédita, en la que se realiza un estudio sobre la inmigración desde el punto de vista antropológico. Gabriela SCHIAVONI en Colonos y ocupantes (1998), se aboca al estudio de la ocupación en la frontera agraria a través del parentesco y la reciprocidad. Por su parte, enLos colonos de Misiones, Horacio BELASTEGUI (2004) describe los antecedentes colonizadores, se focaliza en la entrega de la tierra pública y las medidas oficiales para fomentar la colonización y expone las distintas «familias colonizadoras», como son la brasileña, la alemana, la polaca, la ucraniana, entre otras. Las Jornadas Sobre Poblamiento, Colonización e Inmigración de Misiones realizadas por el Instituto Antonio Ruiz de Montoya constituyen un multifacético aporte en lo que respecta al tema (AA.VV. 1999, 2001, 2003, 2005, 2007 y 2009). Otros trabajos sobre grupos migratorios que sirven como antecedente para alemanes (MICOLIS, 1973; MEDING, 1995; KEGLER, 2006), alemanes-brasileños (GALLERO, 2009 a; GALLERO 2010 y GALLERO 2012), escandinavos (FOGELER, 2007), japoneses (YAMAGUCHI, 2000), guaraníes (GOROSITO KRAMER, 1982 y CEBOLLA BADIE, 2016), paraguayos (NIELLA, 2002), brasileños (MENENDEZ, 1971; GALLERO, 2016), polacos en Wanda y Lanusse (KRAUSTOFL, 2007), ucranianos (SNIHUR, 1997), suizos (GALLERO, 2008 y 2009 b). Dichos aportes, entre otros, contribuyen a la comprensión de cada grupo, aunque no profundicen sobre el entramado étnico, sociocultural y fronterizo de la provincia.

Desdeesta perspectiva, la elaboración de una cartografía etnográfica como metodología se fue desarrollando como un desafío que permitiógenerar un gran potencial, como trataremos de demostrar en el apartado siguiente.

\section{LA CARTOGRAFÍA ETNOGRÁFICA APLICADA}

La combinación entre permisión e interdicción, para estudiar el proceso de poblamiento de Misiones, fue abriendo senderos y marcando un horizonte, en el cual el hallazgo de fuentes, además de ser un reto permanente, significó repensar cómo se trabajaría el material hallado.

Un antecedente histórico de la provincia,que se debe tener en cuenta es que al finalizar la Guerra de la Triple Alianza (1865-1870), la provincia de Cor- 
rientes ejerció el dominio y usufructo de las riquezas naturales. Posteriormente, pasó a depender del gobierno nacional con la creación del Territorio Nacional de Misiones (1881) y a contar conuna estructura administrativa propia. Durante el proceso de federalización, se generó una dura polémica con el gobierno nacional cuando se presentó el proyecto de Ley sobre la Federalización de Misiones. Sin embargo, cuando en la legislatura correntina se percataron que el proyecto se llevaría a cabo de todasmaneras - y que de ese modo perderían el usufructo de la tierra-, autorizaron la enajenación de los departamentos de Candelaria y San Javier, los únicos que existían por entonces, extendiéndose hasta el territorio litigioso que posteriormente se perdió ante Brasil. En esta venta, que se ha dado a conocer como «la noche triste de Misiones», se repartieron más de 762 leguas cuadradas entre 29 personas, una superficie equivalente a 2101936 hectáreas del territorio de ese entonces (las dos terceras partes de lo que sería la provincia) (STEFAÑUK, 1999: 304).Dicha operación dejó una impronta negativa en la estructura territorial, pues dio origen a grandes latifundios y, como consecuencia de ello, la colonización oficial tuvo que avanzar a lo largo de la franja central del espacio misionero.

A partir de ese momento, y por casi cuatro décadas, la colonización fue propiciada por el Estado, con la característica de oficializar colonias que habían surgido por la afluencia espontánea de pobladores. Oberá, fundada en 1927, es la colonia más representativa de esta modalidad. A la inmigración le cupo el rol de poblar un territorio que estaba «vacío» para los cánones de civilización europea. Fueron distintos grupos migratorios - entre los que se destacan paraguayos, brasileños, polacos, ucranianos, escandinavos, alemanes, suizos, españoles e italianos - que se sumaron al proyecto colonizador. Otras colonias oficiales que se afianzaron fueron Apóstoles, Azara, Candelaria, Aristóbulo del Valle, entre otras. Su clima benigno,su vegetación exuberante y sus suelos fértilesse consideraron ideales para desarrollar proyectos colonizadores.

A fines de 1919 entraron en escena las compañías privadas y se concretaron dos proyectos colonizadores de gran envergadura. Uno liderado por Adolfo Schwelm con la fundación de Eldorado y el otro, por Carlos Culmey, quien fundó Puerto Rico y Montecarlo. Estas colonias ubicadas sobre la ribera del Alto Paraná serían finalmente administradas por la «Compañía Colonizadora Eldorado, Explotación de Bosques Ltda.» (véase la Figura 1).

Figura 1 - Plano con las colonias de la Compañía Colonizadora Eldorado

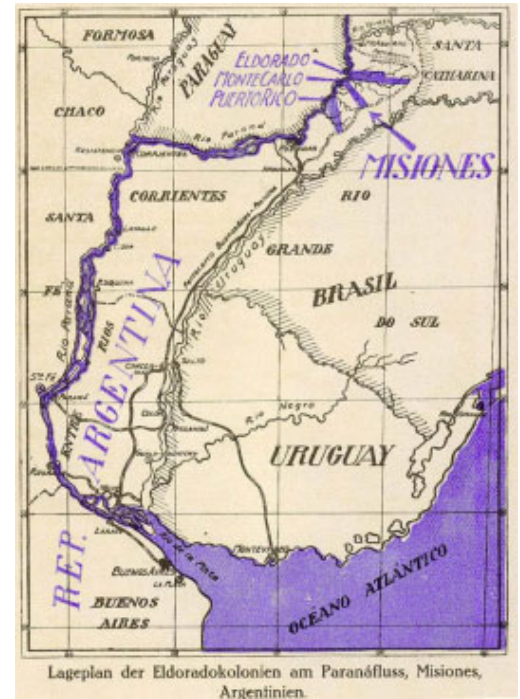

Fuente: Die Eldorado-Kolonien am Paraná Fluss, 1925, p. 57. 
Estas empresas pudieron desarrollar proyectos colonizadores en los extensos fundos que dominaban la mayor parte del territorio misionero (EIDT, 1971). Así, la colonización privada le dio un impulso final y decisivo a la colonización estatal, puesto que por un lado, permitió el ingreso de colonos a las tierras que estaban vedadas al accionar oficial y, por otro lado, ocupó un territorio físicamente inaccesible — dominado por la selva — que había frenado todo intento de ocupación permanente hasta ese momento.

En el caso de la Compañía Eldorado, la empresa particular de mayor envergadura, intervino activamente en la institución del dispositivo mercantil relativo a la tierra, orientándose hacia una demanda específica: pobladores de origen europeo, mayoritariamente alemanes y alemanes-brasileños, con cierta capacidad de pago. La variable sobre el origen étnico evidenció que la Compañía Eldorado fue exitosa en determinar el asentamiento de inmigrantes germánicos, quienestendrían influencia a largo plazo en las relaciones interétnicas con los criollos, indígenas o agentes del estado nacional (GALLERO y CEBOLLLA BADIE, 2011 y 2016).

Por consiguiente, las colonias de Puerto Rico, Montecarlo y Eldorado fueron consideradaspor mucho tiempo como asentamientos destinados a los colonos alemanes y sus descendientes. Los principales folletos propagandísticos se redactaban exclusivamente en idioma alemán, lo que pone de manifiesto la direccionalidad y exclusividad del tipo de inmigrante buscado ${ }^{2}$.

La mediación monetaria y los preliminares expertos (mensura) constituyeron a la tierra como mercancía. Esta estructuración resultó acertada porque la tierra fiscal disponible no era de calidad equiparable ni muy abundante y no ofrecía las garantías de una pronta escrituración (GALLERO, 2009 a; y GALLERO y SCHIAVONI, 2017).

\section{ACERCA DE LA METODOLOGÍA DE LA CARTOGRAFÍA ETNOGRÁFICA}

La cartografía etnográfica como «artefacto cultural» (PICCOLOTTO, 2004: 193) fue ideada para representar la espacialidad de las relaciones sociales $\mathrm{y}$, en calidad de construcción y discurso se constituye en un «arte de persuasión» (HARLEY, 2005: 63).

El acceso al archivo privado de la Compañía Colonización y Explotación de Bosques Eldorado - ubicado en la ciudad homónima - fue fundamental para analizar el proceso de poblamiento, construir y demarcar una cartografía etnográfica que diera cuenta de esta espacialidad.Dicha Compañía conserva ordenadamente todos los datos relativos a la venta de los terrenos, a saber: el libro de registro de ventas, las carpetas con los boletos de compraventa, y los ficheros donde se consignan los datos de los lotes vendidos. Con esta información se programó la interfaz del programa File Maker Pro y, en la respectiva base de datos, se cargó la siguiente información: lugar, número de lote, superficie, nombre y apellido del comprador, fecha de venta, fecha de escrituración, precio e importe total, transferencias, nombre y apellido de quien lo escrituró y, en algunos casos, los datos de la escritura.Esta matriz de datos luego se analizó conel programa estadístico Excel. Se debe tener en cuenta que los datos estadísticos aluden a características relativas a unidades concretas que componen una población o universo, en este caso, la información correspondiente a cada lote vendido por la Compañía. 
Luego, para poder analizar el proceso de ocupación se consideraron tres variables: la fecha de compra del terreno, su ubicación y el origen étnico del comprador. Además, cada variable se organizó en categorías, una vez quetodo dato hace referencia a una estructura constituida por tres elementos: unidad de análisis, variable y valor (BARANGER, 1999).

Para la primera variable, se tuvo en cuenta la fecha en que el comprador adquirió el terreno ${ }^{3}$. Cabe resaltar que no siempre este dato significa la ocupación efectiva del terreno, pero sí es un indicador válido para analizar la ocupación como proceso, y por otra parte, inferir la llegada de los inmigrantes a la nueva colonización. La fecha de compra fue reducida al nivel ordinal de medición agrupándola por décadas, salvo en el caso de la primera década (1920), que a su vez se dividió en dos quinquenios. Esta división se realizó, por un lado, para poder distinguir entre las ventas realizadas por la Compañía Colonizadora Alto Paraná (1919-1924) y por la Compañía Eldorado (1925 al presente); y por otro lado, para poder analizar más profundamente la llegada de los primeros inmigrantes. El corte temporal que se analizó fue 1919-1969; la primer fecha (1919) corresponde al inicio de la colonización, mientras que el cierre marca el fin de la disponibilidad de los lotes y la saturación de la tierra en la colonia ${ }^{4}(1969)$ (véase el Cuadro 1).

Una aclaración importante es que al observar la cantidad de lotes de cada Colonia, Puerto Rico sobresale por poseer las secciones «Puerto Rico Solares», «San Alberto Pueblo» y «Puerto Rico Pueblo Quintas», las cuales suman casi el $35 \%$ de los lotes vendidos por la compañía, lo que desbalancea la comparación con las otras colonias por carecer de un fichado similar sobre sus secciones urbanas (Compárense loscuadros 2, 4 y 6).

Cuadro 1 - Venta de lotes en Montecarlo, Puerto Rico y Eldorado (1919-1969)

\begin{tabular}{|c|c|c|c|c|c|c|}
\hline & \multicolumn{2}{|c|}{ MONTECARLO } & \multicolumn{2}{c|}{ PUERTO RICO } & \multicolumn{2}{c|}{ ELDORADO } \\
\hline Entre 1919-1924 & 205 & $29,7 \%$ & 247 & $11,9 \%$ & 167 & $9,8 \%$ \\
\hline Entre 1925-1929 & 96 & $13,9 \%$ & 217 & $10,5 \%$ & 420 & $24,6 \%$ \\
\hline Entre 1930-1939 & 101 & $14,6 \%$ & 431 & $20,8 \%$ & 366 & $21,4 \%$ \\
\hline Entre 1940-1949 & 140 & $20,3 \%$ & 484 & $23,3 \%$ & 413 & $24,2 \%$ \\
\hline Entre 1950-1955 & 118 & $17,1 \%$ & 158 & $7,6 \%$ & 249 & $14,6 \%$ \\
\hline Entre 1960-1969 & 30 & $4,3 \%$ & 538 & $25,9 \%$ & 95 & $5,6 \%$ \\
\hline & 690 & $100 \%$ & 2075 & $100 \%$ & 1710 & $100 \%$ \\
\hline
\end{tabular}

Fuente: Elaboración personal.

En la segunda variable se tuvo en cuenta la organización de la propia compañía para la ubicación de los terrenos vendidos, como se verá en el apartado referentea cada colonia.

Para la tercera variable, origen étnico,se tuvo en cuenta el apellido, y en el caso de conocerse, la procedencia del comprador. La «dimensión simbólica de etnicidad», propuesta por Giralda Seyferth, fue útil para ir dibujando una cartografía que permitiera identificar el origen nacional o étnico (SEYFERTH, 2000). Así, por ejemplo, para el caso de la migración alemana-brasileña, se tuvieron que rastrear las implicancias de la germaneidad, y luego convertirlasen una herramienta de análisis. De modo que, al igual que un periscopio permite observar los objetos exteriores cuando están frente a un obstáculo, la identificación de las personas de origen germano [Deutsch] obligó a reclasificarlas para poder hacerlas visibles. Ciudadanos brasileños, argentinos, paraguayos o rusos fueron «desenmascarados» para descubrir una identidad que estaba oculta tras la ciudadanía jurídica ${ }^{5}$. Este método complementario a la cartografía etnográfica, 
denominado como el «periscopio de la germaneidad», se utilizó para identificar el origen étnico de los compradores de lote en el proceso de ocupación de la Colonia Puerto Rico, Montecarlo y Eldorado.

La cartografía etnográfica comenzó a elaborarse manualmente en la Colonia Puerto Ricocon base en el mapa de la Compañía, en el cual pacientemente se coloreó cada lote según la identificación del origen étnico del comprador. Esta primera experiencia fue favorable para tratar de idear una cartografía etnográfica georreferenciada cuando se amplió la base de datos a las colonias de Montecarlo y Eldorado. Es decir, se vinculó la base de datos creada a una base georreferenciada del parcelado catastral, lo que resultó en un SIG (Sistema de Información Geográfica) que permitió elaborar los mapas. En este paso se contó con la colaboración y asistencia técnica del Ingeniero Forestal Abel Scherf, especializado en SIG y Teledetección dedicado a prestar servicios en georreferenciamiento.

Este proceso de vinculación de SIG-EXCEL se inició con los insumos necesarios: mapas históricos de referencia (citados en fuentes cartográficas en la sección bibliografía), catastro digital (como plantilla), software (Programa Qgis); y la base de datos con la información histórica, creadaen File Maker Pro, y luego transferida a Excel. Dicho proceso se dividió en seis pasos. En el primero, se seleccionaron y se reconstruyeron digitalmente los lotes sobre el catastro actual, para lo cual se tuvieron en cuenta mapas históricos como referencia. En el segundo, se cargó,en los lotes seleccionados, un código de lote constituido por el nombre de la línea, el número y la fracción correspondientes. En el tercero, se construyó el mismo código de lote del SiGen la base de datos Excel. En el cuarto, se procedió a vincular la información georreferenciada ( $\mathrm{SiG})$ con la base de datos (Excel) usando el código de lote como patrón de vinculación. En el quinto, se chequeó el resultado de la vinculación. Por fin, en el sexto paso, se confeccionaron los mapas en los que se graficó la información contenida en la base de datos recientemente vinculada.

A continuación, seda cuenta de la respectiva «cartografía etnográfica» de cada una de las colonias estudiadas.

\section{EL POBLAMIENTO DE PUERTO RICO}

Puerto Rico fue fundada por la Compañía Colonizadora Alto Paraná en el año 1919. Los gestores de esta colonización fueron Carlos Culmey, el padre Max von Lassbergs.j. y el administrador Federico Guillermo Rauber, quienes tenían experiencia colonizadora y conocían las particularidades y ventajas delos colonos alemanes-brasileños al haber pasado por «la escuela de la selva brasileña»fueron claves para guiarlos de manera organizada a este territorio. Fue en Puerto Rico donde se concentraron las familias alemanas-brasileñas provenientes de Cachoeira, Santa Cruz, São Sebastião do Caí, São Leopoldo, Santa Maria, Cerro Largo, BomPrincípio, Venâncio Aires, entre otras, ubicadas en el estado brasileño de Rio Grande do Sul.

Para confeccionar la cartografía etnografía sobre Puerto Rico, se utilizó como base el plano de Colonias Puerto Rico y San Alberto (c. 1920), en el que se encuentra ubicadocada lote en el fraccionamiento según la denominación de una línea o sección, por ejemplo: Línea Paraná, Línea Capioví, San Alberto Sección Primera, etc. (Primera y segunda variable) (Cuadro 2). 
Cuadro 2 - Lotes vendidos por período según su ubicación en la Colonia Puerto Rico

\begin{tabular}{|l|c|c|c|c|c|c|c|}
\hline $\begin{array}{c}\text { PERÍODO } \\
\text { FRACCIONES }\end{array}$ & $\mathbf{1 9 1 9 /}$ & $\mathbf{1 9 2 5 /}$ & $\mathbf{1 9 3 0 /}$ & $\mathbf{1 9 4 0 /}$ & $\mathbf{1 9 5 0 /}$ & $\mathbf{1 9 6 0 /}$ & $\begin{array}{c}\text { TOTA- } \\
\text { LES }\end{array}$ \\
\hline Capioví & 55 & 10 & 39 & 7 & 6 & - & 117 \\
\hline Cuña Pirú Pueblo & 19 & 5 & 9 & 21 & 2 & - & 56 \\
\hline Cuña Pirú Quintas & 9 & 17 & 27 & 35 & - & 1 & 89 \\
\hline Cuña Pirú Sec. II & - & 1 & - & 3 & 78 & 28 & 110 \\
\hline Línea Cuchilla & 9 & 9 & 87 & 55 & 1 & - & 161 \\
\hline Línea León & 17 & 17 & 24 & 3 & 1 & - & 62 \\
\hline Línea Mbariguí & 2 & 6 & 24 & 7 & - & - & 39 \\
\hline Línea Mbopicuá & 17 & 13 & 19 & 3 & 1 & - & 53 \\
\hline Línea Paraná & 16 & 4 & 5 & 8 & - & - & 33 \\
\hline Línea Tucangua & 13 & 2 & 6 & 36 & 3 & - & 60 \\
\hline $\begin{array}{l}\text { Puerto Rico } \\
\text { Quintas }\end{array}$ & - & 0 & 8 & 30 & 3 & - & 41 \\
\hline $\begin{array}{l}\text { Puerto Rico } \\
\text { Pueblo Quintas }\end{array}$ & 36 & 45 & 21 & 19 & 1 & 2 & 124 \\
\hline $\begin{array}{l}\text { Puerto Rico } \\
\text { Solares }\end{array}$ & 2 & 5 & 26 & 75 & 44 & 316 & 468 \\
\hline San Alberto Sec. I & 1 & 13 & 35 & 6 & - & 1 & 56 \\
\hline $\begin{array}{l}\text { San Alberto Sec. } \\
\text { I A }\end{array}$ & - & - & - & 2 & - & 43 & 45 \\
\hline $\begin{array}{l}\text { San Alberto Sec. } \\
\text { II }\end{array}$ & 17 & 17 & 26 & 82 & 6 & - & 148 \\
\hline $\begin{array}{l}\text { San Alberto Sec. } \\
\text { II Quintas }\end{array}$ & 13 & 43 & 47 & 2 & - & 15 & 120 \\
\hline $\begin{array}{l}\text { San Alberto Sec. } \\
\text { III }\end{array}$ & 20 & 10 & 28 & 90 & 12 & 4 & 164 \\
\hline $\begin{array}{l}\text { San Alberto } \\
\text { Pueblo }\end{array}$ & 1 & - & - & - & - & 128 & 129 \\
\hline Totales & 247 & 217 & 431 & 484 & 158 & 538 & 2075 \\
\hline
\end{tabular}

Para la tercera variable de «origen étnico» se organizaron las siguientes categorías: «alemán-brasileño» [Deutschbrasilianer] abarcó a toda persona con ascendencia alemana, pero cuya nacionalidad es brasileña. Aquí también se incluyeron a sus descendientes, es decir, alemanes-brasileños nacidos en Misiones. «Alemán» [Deutsch] englobó a todo individuo con este origen, como los alemanes del Volga, los austríacos o los alemanes de Alemania. «Suizo»comprende a toda persona de dicha nacionalidad (incluye suizos que hablan dialecto suizo-alemán, francés e italiano). «Criollo» denomina a todo individuo de origen hispano, sin que se pueda distinguir si son argentinos, paraguayos o de otra nacionalidad. «Otros» incluye a los individuos cuyo origen es difícil descifrar - no fue posible diferenciar apellidos polacos, ucranianos o rusos-, o que por su poca representación no se consideran una categoría aparte - franceses, italianos, ingleses-. En esta categoría también se incluyen los lotes destinados a los organismos oficiales, las plazas, el obispado o la gendarmería (Cuadro 3). 
Cuadro 3 - Lotes vendidos por período y origen étnico en la Colonia Puerto Rico

\begin{tabular}{|c|c|c|c|c|c|c|}
\hline & $\begin{array}{c}\text { Alema- } \\
\text { nes-brasi- } \\
\text { leños }\end{array}$ & Alemanes & Suizos & Criollos & Otros & Totales \\
\hline $\mathbf{1 9 1 9 / 1 9 2 4}$ & 184 & 33 & 4 & 25 & 1 & $\mathbf{2 4 7}$ \\
\hline $\mathbf{1 9 2 5} / \mathbf{1 9 2 9}$ & 151 & 21 & 13 & 11 & 21 & $\mathbf{2 1 7}$ \\
\hline $\mathbf{1 9 3 0} / \mathbf{1 9 3 9}$ & 243 & 31 & 123 & 16 & 18 & $\mathbf{4 3 1}$ \\
\hline $\mathbf{1 9 4 0} / \mathbf{1 9 4 9}$ & 313 & 66 & 54 & 26 & 25 & $\mathbf{4 8 4}$ \\
\hline $\mathbf{1 9 5 0} / 959$ & 57 & 41 & 5 & 28 & 27 & $\mathbf{1 5 8}$ \\
\hline $\mathbf{1 9 6 0} / \mathbf{1 9 6 9}$ & 144 & 37 & 18 & 308 & 31 & $\mathbf{5 3 8}$ \\
\hline Totales & $\mathbf{1 0 9 2}$ & $\mathbf{2 2 9}$ & $\mathbf{2 1 7}$ & $\mathbf{4 1 4}$ & $\mathbf{1 2 3}$ & $\mathbf{2 0 7 5}$ \\
\hline & $52,6 \%$ & $11 \%$ & $10,5 \%$ & $20 \%$ & $5,9 \%$ & 100 \\
\hline
\end{tabular}

Fuente: Elaboración personal.

El análisis de esta variable, desde el inicio de la colonización hasta el agotamiento de los lotes coloniales (1919-1969), evidenció la preeminencia de colonos alemanes-brasileños y alemanes en un primer momento, y suizos posteriormente: el porcentaje es de alemanes $11 \%$, alemanes-brasileños de $50,6 \%$, de suizos 10, $5 \%$. En este período, el porcentaje de«otros»-migrantes de diferentes nacionalidades, como franceses, polacos o checoslovacos- fue minoritario, ya que no pasó del 5,9\%; mientras que el porcentaje de «criollos» alcanzó el $20 \%$, quienes se asentaron especialmente en la zona urbana de esta colonia. Guido Rauber y Leonor Kuhn, intrigados por el porcentaje de alemanes plasmado en este cuadro (GALLERO, 2009 a: 147), realizaron una investigación para identificar a los inmigrantes de esta nacionalidad en dos publicaciones tituladas Alemanes de Alemania en los inicios de la colonización de Puerto Rico (RAUBER y KUHN, 2012 y 2014).

En los mapas 1, 2 y 3, que han sido diagramados manualmente ${ }^{6}$, se puede observar un primer resultado de la cartografía etnográfica sobre la Colonia de Puerto Rico. Mientras los dos primeros muestran la distribución de los colonospor período, el tercero muestra la colonia en su totalidad.

Asimismo, en este proceso de asentamiento hay que destacar la manera en que se aplicó el modelo Waldhufen[aldea o caserío con terrenos cultivables en el bosque] (EIDT, 1971). Un sistema de parcelamiento que fue clave para desarrollar la colonización en un medio selvático como lo es la Selva Paranaense o Mata Atlántica. Lo que se pudo constatar, es que para seguir este modelo de parcelamiento, la compañía asentó a quince familias en su propio terreno cultivable comunicadas por una picada, arteria vital para sobrevivir en el monte (véase el Mapa 1). 
Mapa 1 - Origen étnico de los compradores en la Colonia Puerto Rico (1919-1924)

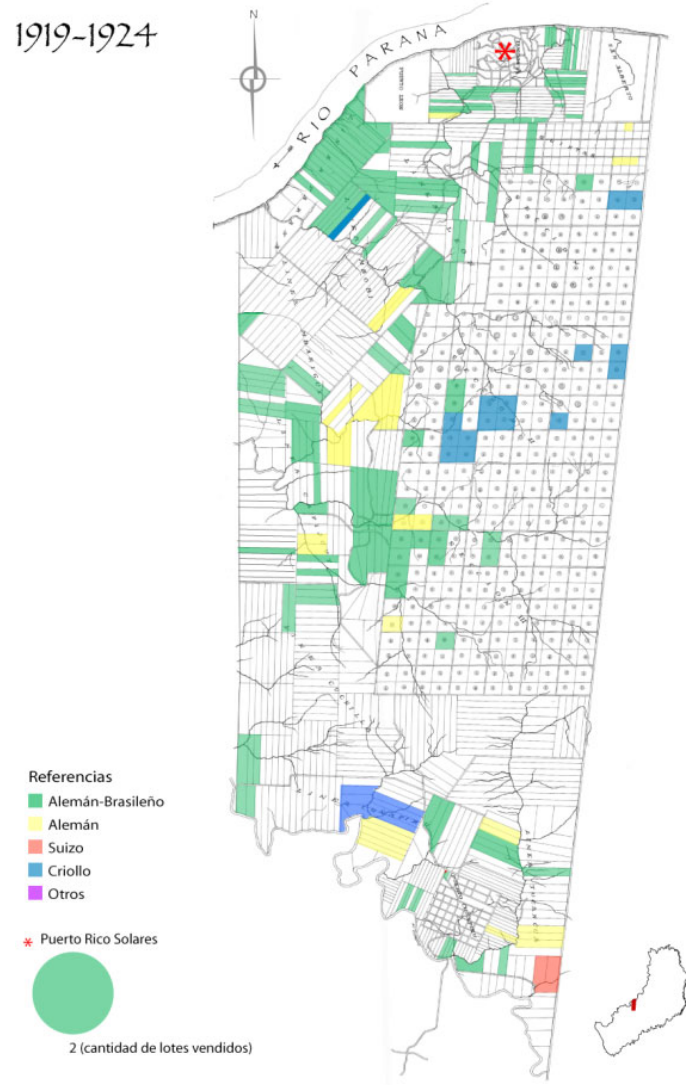

Mapa 2 - Origen étnico de los compradores en la Colonia Puerto Rico (1930-1939)

\section{4}

N

选

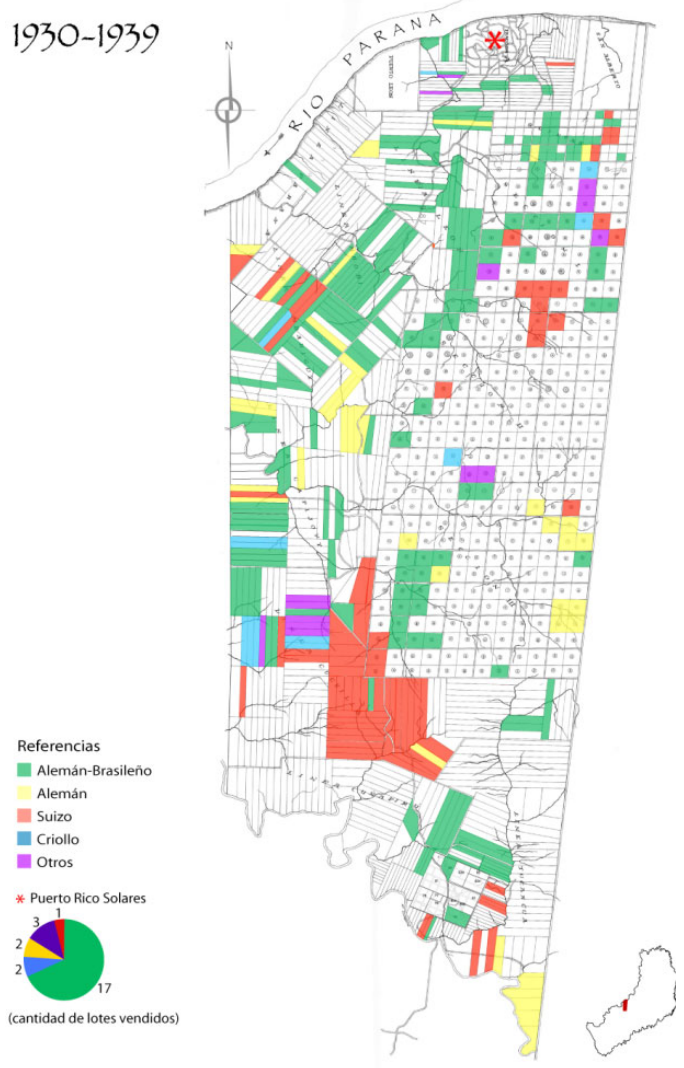

n. $52|2018|$ p. $14-39$ 


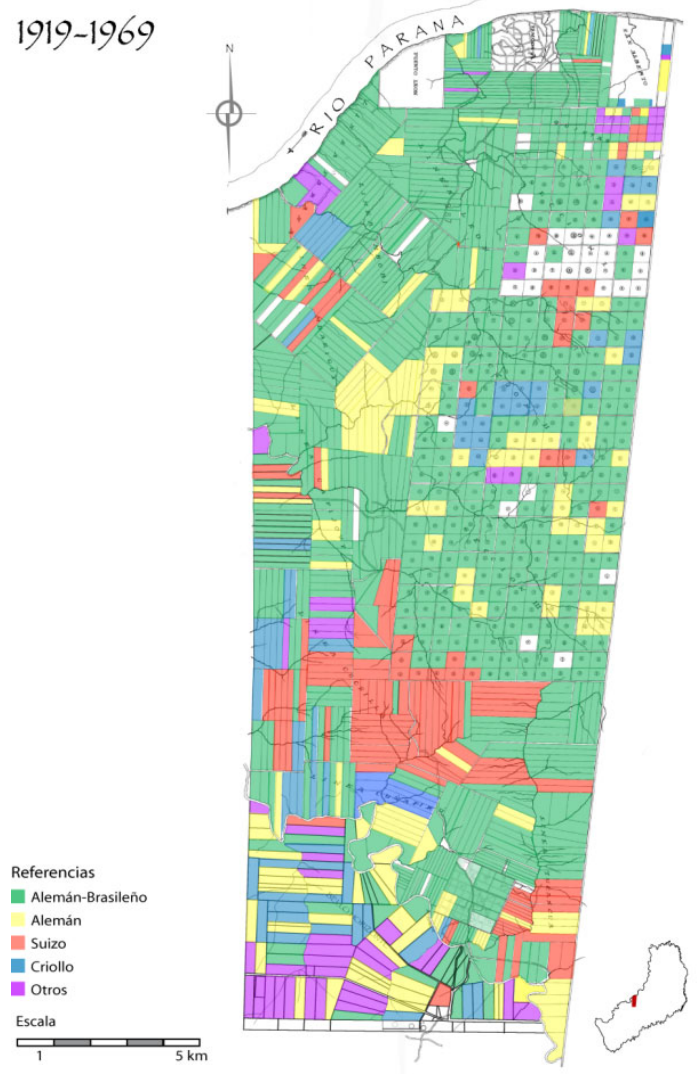

\section{EL POBLAMIENTO DE MONTECARLO}

El plan colonizador que había concretado la fundación de la Colonia Puerto Rico para inmigrantes católicos (1919), al año siguiente, destinó colonos protestantes a Montecarlo (1920). En un principio mantuvo la división religiosa de las colonias y realizó mucha propaganda para atraernuevos colonos, lo cual se reforzó cuando se realizó la fusión de estas colonias con la Compañía Eldorado en 1925, aunque la división religiosa ya no fuera tan tajante.

Thomas Kopp afirma que Culmey, además de asentar a la gente «severamente separada por confesión», pensaba que en las nuevas colonias se deberían admitir solamente alemanes que hubieran «pasado la escuela de la selva en Brasil. De los recién llegados de Alemania no se podía contar con la fortaleza para el rápido desarrollo de las colonias» (KOPP, 1949: 189). Las dificultades de iniciar una colonización en la selva eran grandes y bien conocidas por quienes la dirigían, no en vano el padre Lassberg destinó su relato de viaje «a colonos alemanes-brasileños que se inclinan y necesitan emigrar», quienes tenían como «misión formar la base de ayuda y sostén para los recién llegados de Alemania en las nuevas colonias» (citado en GALLERO, 2009 a: 86).Desdeesta visión, lo importante es formar parte de la «comunidad alemana», en donde lo fundamental es la ascendencia germana [Deutschstämmigkeit].

Esta colonia también fue parcelada siguiendo el modelo Waldhufen, por lo cual se asentaron familias primero en Línea Aterrada, Línea Bonita y Montecarlo Quintas y, a partir de 1925, en Línea Guatambú, Línea Caraguatay e Itacuruzú.Para analizar el poblamiento de Montecarlo se realizó el mismo trabajo con la base de datos y se utilizaron las mismas variables, aunque con 
una diferencia importante, que fue realizar la cartografía con base en elgeorreferenciamiento y trabajar con el programa «Qgis». La cartografía etnográfica se realizó con base en el plano de Colonia Montecarlo (c. 1920), en el que se ubicaron los lotes según el parcelamiento realizado por la Compañía (primera y segunda variable) (Cuadro 4).

Cuadro 4 - Lotes vendidos por período según su ubicación en la Colonia Montecarlo

\begin{tabular}{|c|c|c|c|c|c|c|c|}
\hline $\begin{array}{c}\text { PERÍODO } \\
\text { FRACCIO- } \\
\text { NES }\end{array}$ & $\begin{array}{l}1919- \\
1924\end{array}$ & $\begin{array}{l}1925- \\
1929\end{array}$ & $\begin{array}{l}1930- \\
1939\end{array}$ & $\begin{array}{l}1940- \\
1949\end{array}$ & $\begin{array}{l}1950- \\
1959\end{array}$ & $\begin{array}{l}1960- \\
1969\end{array}$ & $\begin{array}{l}\text { TO- } \\
\text { TA- } \\
\text { LES }\end{array}$ \\
\hline $\begin{array}{l}\text { Línea Alto } \\
\text { Itacuruzú }\end{array}$ & & & 24 & 19 & 13 & 2 & 58 \\
\hline $\begin{array}{c}\text { Línea } \\
\text { Aterrada }\end{array}$ & 21 & 10 & 6 & & 1 & & 38 \\
\hline $\begin{array}{l}\text { Línea } \\
\text { Bonita }\end{array}$ & 85 & 19 & 10 & 2 & 3 & 0 & 119 \\
\hline $\begin{array}{c}\text { Línea } \\
\text { Caraguatay }\end{array}$ & 22 & 24 & 9 & 15 & 5 & 3 & 78 \\
\hline $\begin{array}{l}\text { Línea } \\
\text { Chica }\end{array}$ & 7 & 7 & 5 & 4 & & 0 & 23 \\
\hline $\begin{array}{c}\text { Línea } \\
\text { Guatambú }\end{array}$ & 15 & 13 & 8 & 12 & 2 & 2 & 52 \\
\hline $\begin{array}{c}\text { Línea } \\
\text { Horqueta II }\end{array}$ & & & & 31 & 81 & 19 & 131 \\
\hline $\begin{array}{c}\text { Línea } \\
\text { Itacuruzú }\end{array}$ & 8 & 1 & 4 & 13 & 4 & 0 & 30 \\
\hline $\begin{array}{c}\text { Montecarlo } \\
\text { Quintas }\end{array}$ & 34 & 4 & 4 & 9 & & & 51 \\
\hline $\begin{array}{c}\text { Paraná } \\
\text { Montecarlo }\end{array}$ & & 8 & & 6 & 1 & & 15 \\
\hline $\begin{array}{c}\text { Piray } \\
\text { Montecarlo } \\
\end{array}$ & 12 & 10 & 30 & 24 & 1 & 1 & 78 \\
\hline Montecarlos & 1 & & 1 & 6 & 7 & 3 & 18 \\
\hline Totales & 205 & 96 & 101 & 141 & 118 & 30 & 690 \\
\hline
\end{tabular}

Fuente: Elaboración personal

Para el análisis del origen étnico de Montecarlo, se utilizó como base un trabajo realizado por Juan Plocherque identifica«las diferentes nacionalidades de los pioneros que poblaron Montecarlo» (PLOCHER: 2002). El estudio se realizó con base en un plano que tiene anotado, en cada lote, el nombre de su propietario, lo que permitió enlistar alfabéticamenteal apellidode las 367 familias propietarias de los 450 lotes mensurados hasta ese momento (Mapa 4). 


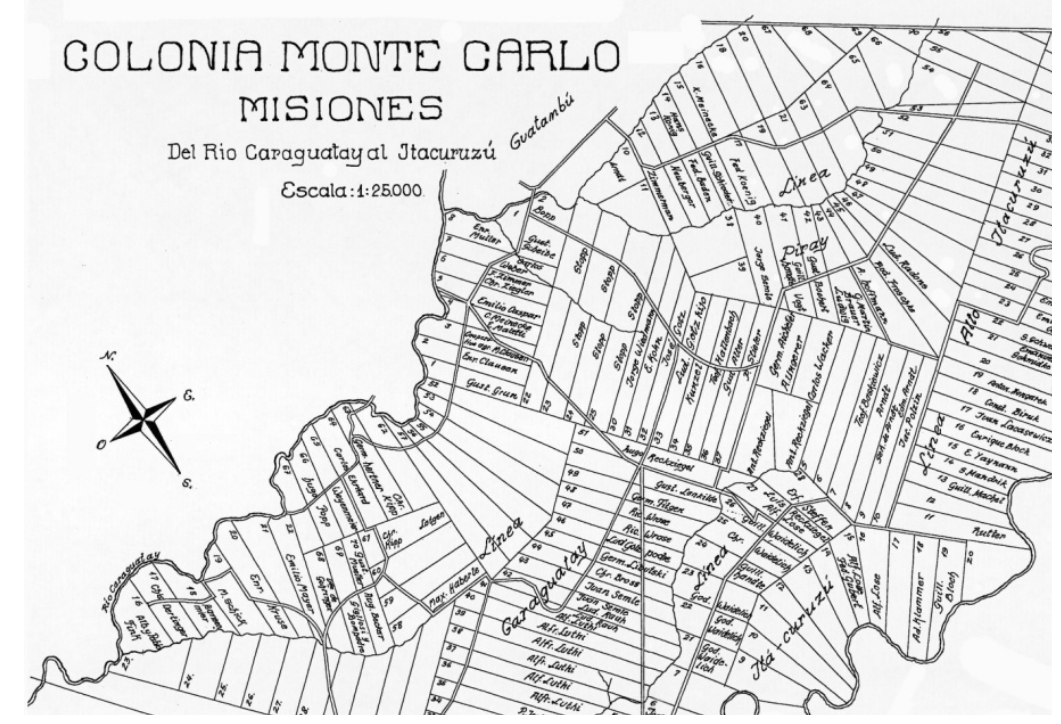

Posteriormente, Juan Plocher recurrió a Emilio Erhardt y Heiner Müller para distinguir el origen de los propietarios ${ }^{7}$. El resultado de esta pesquisa muestra las características poblacionales de esta colonia:

«- De 252 lotes los propietarios son alemanes, lo que representa el $68 \%$, y a su vez, de estos $45 \%$ (114) eran originarios de la provincia de la hoy Baden-Wurttemberg y buena parte de ellos con lotes en la Línea Aterrada.

- De 57 lotes los propietarios son alemanes-brasileños, que representan el $15 \%$.

- De 43 lotes los propietarios son polacos, lo que representa el $12 \%$.

Quiere decir entonces, que el segundo grupo de nacionalidades, después de los alemanes y de los brasileños-alemanes, que se establecieron en Montecarlo, fueron los polacos» ${ }^{8}$ (PLOCHER, 2002: 214).

Cuadro 5 - Lotes vendidos por período y origen étnico en la Colonia Montecarlo

\begin{tabular}{|c|c|c|c|c|c|c|c|c|c|}
\hline &  & 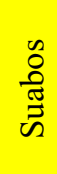 & 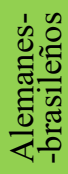 & 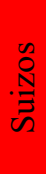 & $\begin{array}{l}0 \\
0 \\
0 \\
0 \\
0 \\
0\end{array}$ & 声告 & 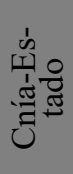 & อี & $\frac{\frac{\theta}{\pi}}{\tilde{\sigma}}$ \\
\hline $\begin{array}{l}1919 / \\
1924\end{array}$ & 79 & 57 & 58 & 6 & 3 & & & 3 & 206 \\
\hline $\begin{array}{l}1925 / \\
1929 \\
\end{array}$ & 53 & 23 & 11 & 2 & 2 & 3 & & 2 & 96 \\
\hline $\begin{array}{l}1930 / \\
1939\end{array}$ & 37 & 15 & 16 & 1 & 29 & & 3 & & 101 \\
\hline $\begin{array}{l}1940 / \\
1949\end{array}$ & 46 & 39 & 22 & 1 & 23 & 2 & 2 & 8 & 143 \\
\hline $\begin{array}{l}1950 / \\
1959\end{array}$ & 23 & 23 & 14 & 1 & 39 & 3 & 3 & 7 & 113 \\
\hline $\begin{array}{l}1960 / \\
1969\end{array}$ & 4 & 15 & 4 & & 2 & 2 & 4 & & 31 \\
\hline
\end{tabular}




\begin{tabular}{|c|c|c|c|c|c|c|c|c|c|}
\hline $\begin{array}{c}\text { Tota- } \\
\text { les }\end{array}$ & 242 & 172 & 125 & 11 & 98 & 10 & 12 & 20 & 690 \\
\hline & $\begin{array}{r}35,1 \\
\%\end{array}$ & $\begin{array}{r}24,9 \\
\%\end{array}$ & $\begin{array}{c}18,1 \\
\%\end{array}$ & $\begin{array}{l}1,6 \\
\%\end{array}$ & $\begin{array}{c}14,2 \\
\% \%\end{array}$ & $\begin{array}{l}1,4 \\
\%\end{array}$ & $\begin{array}{r}1,7 \\
\%\end{array}$ & $\begin{array}{l}2,9 \\
\%\end{array}$ & $\begin{array}{c}100 \\
\%\end{array}$ \\
\hline
\end{tabular}

Fuente: Elaboración personal.

Esta distinción de nacionalidades, realizada por los propios vecinos de Montecarlo, fue ratificada al analizarel origen étnico de la base de datos en el momento de examinar el proceso de poblamiento de las diferentes líneas que conforman la colonia.

En primer lugar, sobresalen los colonos alemanes con un 35,1 \% y suabos con un 24,9\%, los cuales en su conjunto representan el $60 \%$ de «alemanes de Alemania», llama la atención el alto porcentaje desuabos de Baden-Wurttemberg. En segundo lugar, se posicionan los alemanes-brasileños con un 17, $9 \%$; seguidos de los polacos con un $14,2 \%$, entre los cuales la mayoría era«alemanes-polacos», aunque fue difícil identificar los «polacos-polacos» de los «polacos alemanes», una vez que «muchos fallecieron y antes se distinguían por la tonada» ${ }^{9}$. En este período, el porcentaje de los llamados«otros»migrantes de diferentes nacionalidades, como norteamericanos, checoslovacos o daneses - fue minoritario, ya que no pasó del 3,4\%; mientras que el porcentaje de los «criollos» alcanzótan solo el 1,5\% y los lotes destinados al Estado o adquiridos por compañías representaron el 1,7 \% en Montecarlo (Mapa 5).

El Pastor Ernst Heuser, quien atendió a los colonos de Montecarlo y Eldorado en las primeras décadas de la colonización, en sus Memorias sobre Misiones, da un ejemplo de las diferencias intraétnicas que se vivenciaban en la colonia:

«Un suabo de Zwerenberg — más tarde establecido en Montecarlomarchaba en el año 1916 como soldado del frente con sus camaradas por una calle estrecha: era el pueblo de Schöndorf cerca de Munkacz. Delante de ellos iba conversando un grupo de campesinos. A los soldados suabos les pareció poder entender su idioma: "Hablan como los Altensteig" o un pueblo parecido... Y efectivamente eran personas de ese lugar, con los cuales festejaron el reencuentro después de una separación de unos 170 años. Estos recuerdos facilitaron la mutua comprensión, también en las colonias de la selva» (HEUSER, c.1980).

Estas diferencias dialectales se prestan para un profundo análisis lingüístico que excede el objetivo de este artículo. 
Mapa 5 - Origen étnico de los compradores en la Colonia Montecarlo (1919-1924)

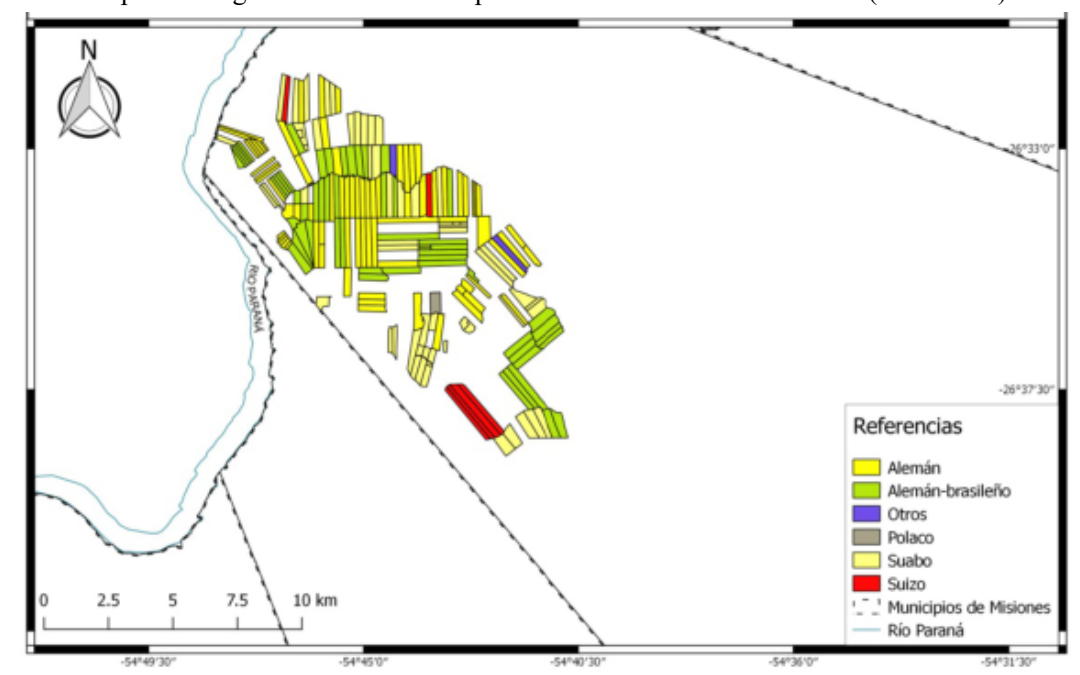

Mapa 6 - Origen étnico de los compradores en la Colonia Montecarlo (1919-1969)

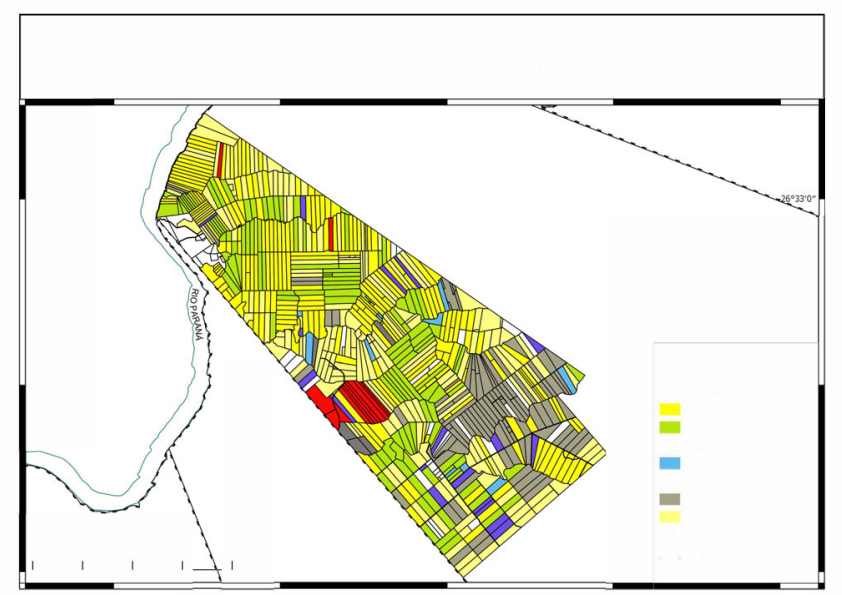

\section{EL POBLAMIENTO DE ELDORADO}

Eldorado se inició cuando Adolfo Schwelm decidió fundar, el 29 de septiembre de 1919, una colonia destinada principalmente a inmigrantes europeos que buscaran un nuevo horizonte tras la devastación que había producido la Primera Guerra Mundial. Por otra parte, Elida Arenhardt plantea que el origen de la organización del espacio geográfico de Eldorado fue resultado del proyecto personal de Schwelm de «poblar y producir», influenciado por el accionar de la Real Sociedad Geográfica de Londres, de la que era socio activo (ARENHARDT, 2003: 156).

Aunque el análisis del proceso de poblamiento de esta colonia se haya realizado del mismo modo que el de Montecarlo, fue más intrincadoporque tenía más Líneas que habían sido mensuradas por diversos agrimensores,y además, la concurrencia de colonos reveló un origen más variopinto que Montecarlo y Puerto Rico.

La cartografía etnográfica se realizó basándose en los mapas y planos de la Colonia Eldorado(1927, 1930, 1939, 1945) (como ejemplo, véase el Mapa 
7).En el Cuadro 6 se puede observar que la extensión de la colonia permitió a la compañía asentar acolonos a medida que se abrían nuevas líneas. Así, en un primer momento (entre 1919-1949) se ocuparon las fracciones denominadas Eldorado, Bence, Chappaz, Pomar, Whitaker y Hasselbach F y G, posteriormente se inició la ocupación de las fracciones I, L, N, O, P, Q, S, T (primera y segunda variable).

Cuadro 6 - Lotes vendidos por período según su ubicación en la Colonia Eldorado

\begin{tabular}{|c|c|c|c|c|c|c|c|}
\hline $\begin{array}{c}\text { PERÍODO } \\
\text { FRACCIO- } \\
\text { NES }\end{array}$ & $\begin{array}{l}1919 / \\
1924\end{array}$ & $\begin{array}{l}1925 / \\
1929\end{array}$ & $\begin{array}{l}1930 / \\
1939\end{array}$ & $\begin{array}{l}1940 / \\
1949\end{array}$ & $\begin{array}{l}1950 / \\
1959\end{array}$ & $\begin{array}{l}1960 / \\
1969\end{array}$ & $\begin{array}{l}\text { TO- } \\
\text { TA- } \\
\text { LES }\end{array}$ \\
\hline Eldorado & 23 & 21 & 3 & 22 & 3 & 13 & 85 \\
\hline Bence & 56 & 12 & 3 & 3 & 1 & & 75 \\
\hline Chappaz & 6 & 31 & 2 & & 1 & & 40 \\
\hline Pomar & 17 & 32 & 3 & & & & 52 \\
\hline Whitaker & 45 & 112 & 27 & 17 & 1 & 2 & 204 \\
\hline Hasselbach F & 12 & 58 & 1 & 1 & & & 72 \\
\hline Hasselbach G & & 122 & 113 & 77 & 9 & & 321 \\
\hline Línea I & 4 & 18 & 83 & 14 & 28 & 3 & 150 \\
\hline Línea L & 1 & 2 & 78 & 44 & & & 125 \\
\hline Línea $\mathrm{N}$ & 3 & 9 & & 9 & & 4 & 25 \\
\hline Línea $\mathrm{O}$ & & 3 & 51 & 88 & 13 & 3 & 158 \\
\hline Línea P & & & & 112 & 32 & 10 & 154 \\
\hline Línea Q & & & 2 & 26 & 39 & 7 & 74 \\
\hline Línea $\mathrm{S}$ & & & & & 114 & 4 & 118 \\
\hline Línea T & & & & & 8 & 49 & 57 \\
\hline Totales & 167 & 420 & 366 & 413 & 249 & 95 & 1710 \\
\hline
\end{tabular}

Fuente: Elaboración personal.

El análisis de la variable de origen étnico fue más complejo y requirió la consulta a la base del Centro de Estudios Migratorios Latinoamericanos ${ }^{10}$. La llegada de inmigrantes de países europeos de diferentes regiones fue abrumadora, por lo quelas diferentes nacionalidades se agrupan bajo familias lingüísticas siguiendo el Atlas de los pueblos de Europa Occidental (SELLIER, 2008: 29). Así, «Escandinavos» representa a daneses, noruegos y suecos con un $4,3 \%$; «eslavos del este» abarca a rusos y ucranianos con un $0,6 \%$; «eslavos del oeste»incluye a checos, eslovacos y polacos con un 21,2\%; y estos últimos representan la gran mayoría; «eslavos del sur» incluye a croatas, búlgaros y eslovenos con un 1,2\%; «lenguas romanas» agrupa a españoles e italianos con un 3,2 \%; y los «anglosajones» o ingleses apenas alcanzaron el 0,8\%. El porcentaje de los grupos que fueron abordados en las otras colonias varió, así los alemanes representan una mayoría con el 40,5\%;los alemanes-brasileños no son muy numerosos, pues no llegan al 4,4 \%; los suizos ascienden a un 7,4 \%; los criollos a un $4,2 \%$ y las tierras adquiridas por Compañías o destinadas al estado a un 5,2\%. A estas categorías se agregó «desconocido», pues nofue posible identificar el origen del comprador en un 6,8 \% (Cuadro 7y Mapa 8). 
Cuadro 7 - Lotes vendidos por período y origen étnico en la Colonia Eldorado

\begin{tabular}{|c|c|c|c|c|c|c|c|c|c|c|c|c|c|}
\hline & 导 & 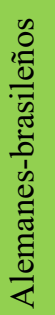 & 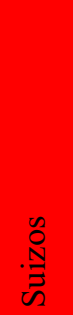 & 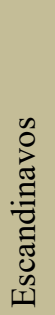 & $\begin{array}{l}\mathscr{D} \\
\mathscr{E} \\
\frac{0}{00} \\
\Xi\end{array}$ & 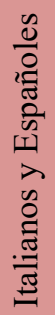 & 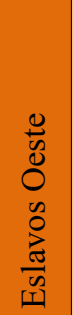 & 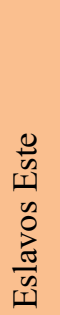 & 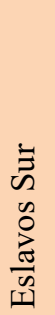 & $\frac{\mathscr{0}}{\stackrel{0}{0}}$ & 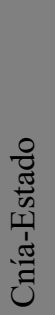 & 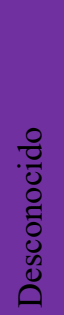 & $\frac{\mathscr{E}}{\stackrel{\sigma}{\frac{\pi}{\theta}}}$ \\
\hline $\begin{array}{l}1919 / \\
1924\end{array}$ & 104 & 11 & 9 & 18 & 2 & 10 & 3 & 1 & 0 & 3 & 4 & 11 & 176 \\
\hline $\begin{array}{l}1925 / \\
1929\end{array}$ & 185 & 15 & 28 & 47 & 10 & 21 & 49 & 2 & 4 & 9 & 5 & 41 & 416 \\
\hline $\begin{array}{l}1930 / \\
1939\end{array}$ & 158 & 7 & 46 & 5 & 0 & 6 & 65 & 5 & 9 & 5 & 31 & 27 & 364 \\
\hline $\begin{array}{l}1940 / \\
1949\end{array}$ & 152 & 14 & 31 & 4 & 1 & 5 & 131 & 1 & 6 & 22 & 24 & 16 & 407 \\
\hline $\begin{array}{l}1950 / \\
1959\end{array}$ & 64 & 20 & 12 & 0 & 0 & 8 & 95 & 1 & 2 & 25 & 5 & 18 & 250 \\
\hline $\begin{array}{l}1960 / \\
1969\end{array}$ & 30 & 9 & 1 & 0 & 1 & 5 & 19 & 0 & 0 & 8 & 20 & 4 & 97 \\
\hline $\begin{array}{l}\text { Tota- } \\
\text { les }\end{array}$ & 693 & 76 & 127 & 74 & 14 & 55 & 362 & 10 & 21 & 72 & 89 & 117 & 1710 \\
\hline & \begin{tabular}{l}
$\partial^{\circ}$ \\
in \\
\multirow{y}{*}{}
\end{tabular} & $\begin{array}{l}\stackrel{0}{+} \\
\dot{\sigma}\end{array}$ & $\begin{array}{l}0^{0} \\
\sigma_{n}\end{array}$ & $\begin{array}{l}\stackrel{2}{*} \\
\stackrel{2}{*}\end{array}$ & $\begin{array}{l}0 \\
\infty \\
0\end{array}$ & $\begin{array}{l}\text { dे } \\
\text { nे }\end{array}$ & 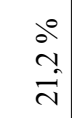 & $\begin{array}{l}0 \\
0 \\
0\end{array}$ & $\begin{array}{l}\text { å } \\
\text { న }\end{array}$ & $\begin{array}{l}\sigma^{0} \\
\stackrel{\gamma}{ }\end{array}$ & $\begin{array}{l}\text { di } \\
\text { ñ }\end{array}$ & $\begin{array}{l}0 \\
\infty \\
0\end{array}$ & $\stackrel{0}{8}$ \\
\hline
\end{tabular}

Fuente: Elaboración personal.

En los mapas 7 y 8 se pretende mostrar la importancia de la primera década para definir los núcleos de poblamiento, mientras en el primer mapa se observan los nombres manuscritos de los compradores y se evidencia una distribución bastante homogénea en lo que hoy es el municipio de Eldorado, en el segundo mapa se observa que la llegada de inmigrantes continuó siendo importante, y debidocasi a susaturación, se dio inicio a la venta de las líneas Hasselbach G, Fracción L y Fracción O, las que serán el Municipio 9 de Julio (1934).

Mapa 7 - Colonia Eldorado (c. 1930)

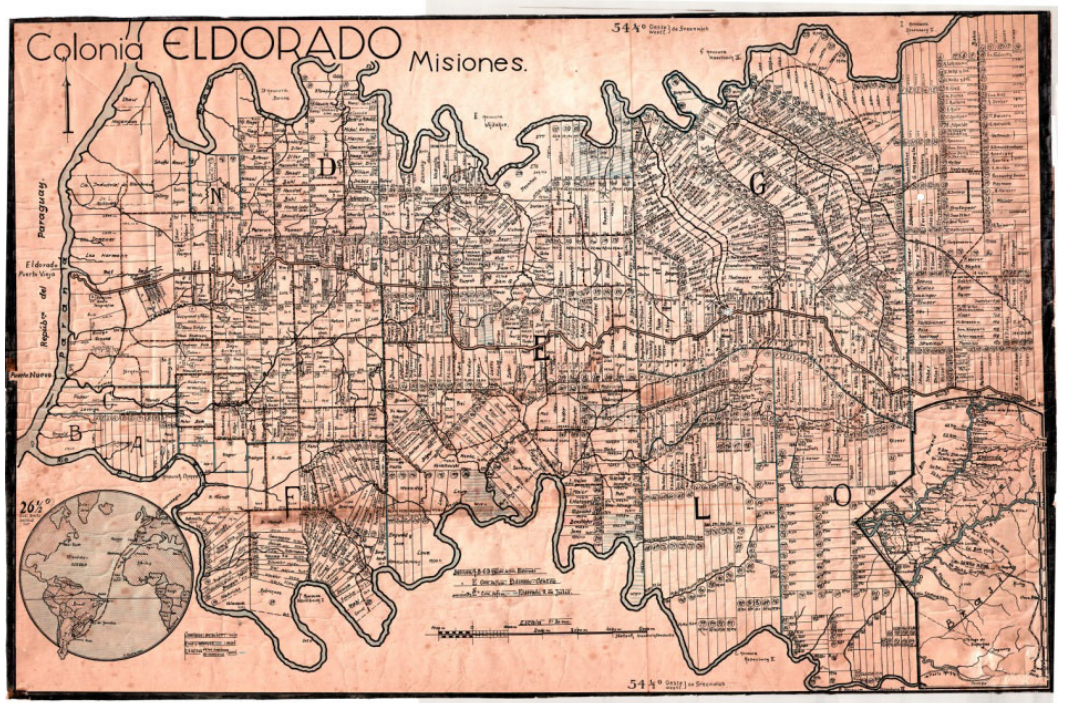


Mapa 8 - Origen étnico de los compradores en la Colonia Eldorado (1925-1929)

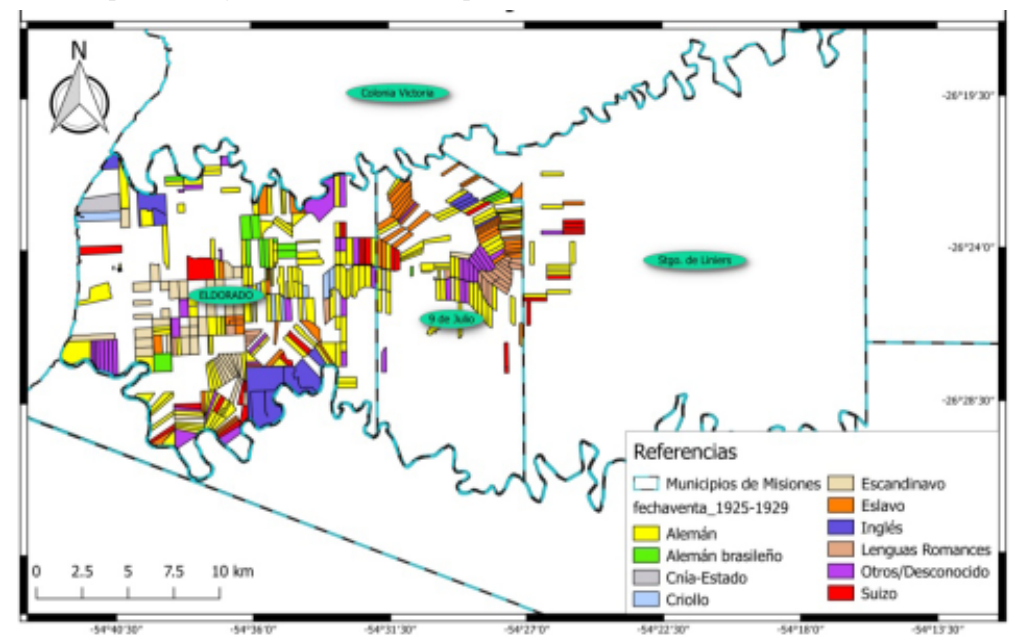

Mapa 9 - Origen étnico de los compradores en la Colonia Eldorado (1919-1969)



Mapa 10 - Fecha de venta de los lotes de la Colonia Eldorado (1919-1989)

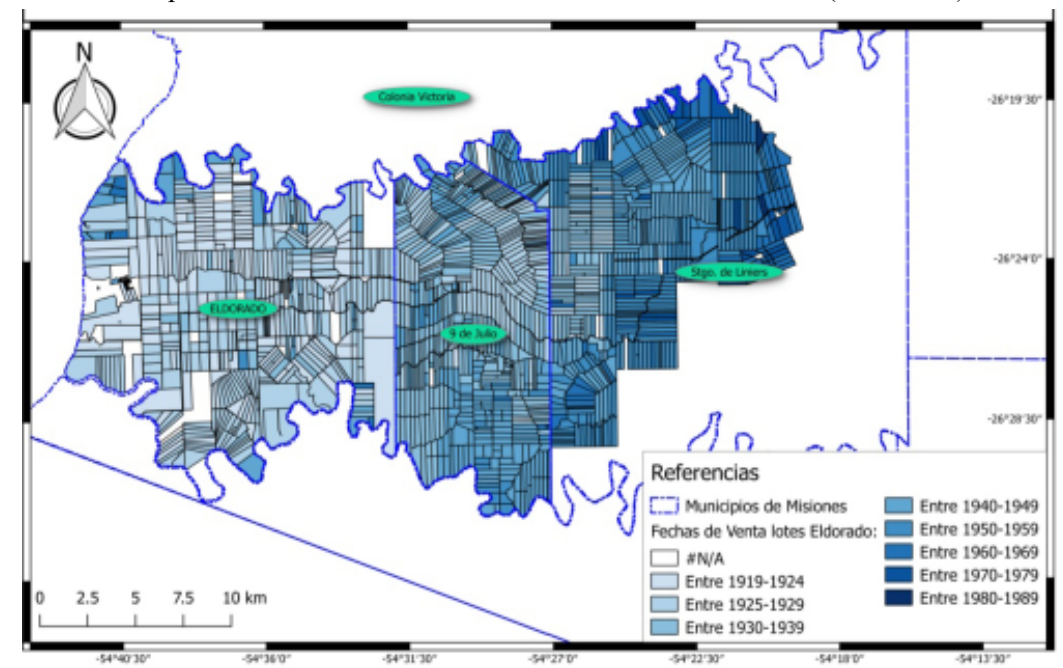

La comparación entre los mapas 9 y 10revela el origen étnico y la graficación temporal de la venta de lotes. Los tonos más claros representan los lotes vendidos más tempranamente; y los más oscuros, los vendidos tardíamente en las fracciones I, P, Q, S (norte y sur) y T, las cuales darán origen al municipio 
de Santiago de Liniers (1947). Estagraficaciónpermite observar aun la manera en que la Compañía loteó y ocupó paulatinamente la tierra desde el río Paraná hasta el interior de Misiones.

\section{REFLEXIONES FINALES}

La cartografía etnográfica como metodología para el estudio del proceso de poblamiento de Misiones reflejó un gran potencial y resultó apropiada - siempre y cuando - hubiera fuentes que permitieran ubicar en un mapa la información recabada.

El hallazgo del archivo de la Compañía Colonizadora y Explotación de Bosques Eldorado fue clave para pensar en cómo analizar la información y en cómo poner en práctica la «observación histórica». La organización de la documentación en una base de datos permitió reconstruir el proceso de poblamientoa partirdelcomprador del lote de tierra:quién y cuándo arribó — aproximadamente - a este territorio.

Por otra parte, las entrevistas y charlas con los informantes, así como los árboles genealógicos y la contrastación de la llegada de los inmigrantes con la base de datos del Centro de Estudios Migratorios Latinoamericanos, fueron claves para reconstruir una etnografía históricay reflejarla enuna cartografía, la cualhasta el momento había dado cuenta del territorio y sus accidentes geográficos, y en algunos casos de los nuevos propietarios (véanse los mapas 4 y 7). Por tanto, la complementación de datos fue esencial para poderasociar posteriormente la base de datos a un sistema georreferenciado, lo que abrió un abanico de posibilidades que da cuenta de cómo se pueden cartografiar el trabajo de archivo y el etnográfico.

Así, como los «mapas redescriben el mundo, al igual que cualquier otro documento, en términos de relaciones y prácticas de poder, preferencias y prioridades culturales» (HARLEY, 2005: 61),los mapas reconstruidos en esta investigación a través de la cartografía etnográfica muestran que los propietarios de las tierras destinadas a cultivo quedaron mayoritariamente en manos de colonos alemanes, alemanes-brasileños, polacos o suizos (Cuadro 8). Esta distribución influyó en las relaciones sociales e interétnicas, en «prácticas de poder», una vez que en un primer momento el grupo criollo trabajó como mano de obra en el desmonte, cosecha de yerba o tung, y luego en diversas ocupaciones en el ramo terciario de los nacientes centros urbanos. Así, mientras los criollos se articularon laboralmente, los indígenas se replegaron hacia el interior de la selva.

Cuadro 8 -Origen étnico de los compradores en Montecarlo y Puerto Rico

\begin{tabular}{|c|c|c|c|c|cc|} 
ORIGEN & \multicolumn{2}{|c|}{$\begin{array}{c}\text { MONTE } \\
\text { ERTICO }\end{array}$} & \multicolumn{2}{|c|}{ PUERTO } & \multicolumn{2}{|c|}{ ELDORADO } \\
RICO & \multicolumn{2}{|c|}{ ELOR } \\
Alemán & 448 & $60,0 \%$ & 229 & $11,0 \%$ & 693 & $40,5 \%$ \\
Alemán-brasileño & 125 & $18,1 \%$ & 1092 & $52,6 \%$ & 76 & $4,4 \%$ \\
Suizo & 11 & $1,6 \%$ & 217 & $10,5 \%$ & 127 & $7,4 \%$ \\
Polaco & 98 & $14,2 \%$ & & & & \\
Criollo & 10 & $1,4 \%$ & 414 & $20,0 \%$ & 72 & $4,2 \%$ \\
Otros/Descono- & 20 & $2,9 \%$ & 123 & $5,9 \%$ & 117 & $6,8 \%$ \\
cidos & 12 & $1,7 \%$ & & & 89 & $5,2 \%$ \\
Cnía-Estado & $12 \%$ \\
Escandinavo & & & & & 74 & $4,3 \%$
\end{tabular}




\begin{tabular}{|c|c|c|c|c|c|c|}
\hline Ingleses & & & & & 14 & $0,8 \%$ \\
\hline$\underset{\text { pañoles }}{\text { Italianos y Es- }}$ & & & & & 55 & $3,2 \%$ \\
\hline Eslavos & & & & & 393 & $23 \%$ \\
\hline Totales & 725 & $100 \%$ & 2075 & $100 \%$ & 1710 & $100 \%$ \\
\hline
\end{tabular}

Fuente. Elaboración personal.

Asimismo, en esta cartografía etnográfica mapear significa conocer a quienes pasaron a controlar y dominar el territorio, dar cuenta de los propietarios que comenzaron«a producir un territorio limitado y continuo sobre una naturaleza discontinua e ilimitada» (PICCOLOTTO, 2004: 230).

En este sentido, el análisis de los datos obtenidos permiten señalar algunas reflexiones generales sobre el proceso de ocupación desarrollado: en primer lugar, los lotes vendidos por la Compañía Colonizadora Alto Paraná en las Colonias de Puerto Rico y Montecarlo; y por Schwelm en Eldorado abrieron una frontera entre el espacio ocupado y la selva (1919-1924). Esta frontera poco a poco fue cediendo paso a la colonización, pero fueron esos primeros núcleos los que determinaron la tendencia de sus respectivos desarrollos. En segundo lugar, el nexo de comunicación entre el puerto y el interior de la colonia fue la picada principal. Mientras la comunicación fue fluvial, la vida de la colonia se volcó hacia sus puertos, pero luego, paulatinamente fue suplantada por la comunicación terrestre, la cualtuvo un impulso decisivo con la creación de la ruta nacional N. ${ }^{\circ} 12$.

Además, los resultados obtenidos permitieron periodizar el proceso de ocupacióna través de la venta de los lotes, como por ejemplo en la Colonia Puerto Rico: en una primera etapa tuvo preeminencia la Compañía Colonizadora Alto Paraná (1919-1924) por ser la rectora de la colonización; luego, tras la reestructuración en la Compañia Eldorado (1925) se inició una segunda etapa que se caracterizó por la consolidación de la colonia y saturación de la tierra (1925-1949) y en la que primó la organización de la vida institucional de la colonia; y finalmente, una tercera etapa en la cual la gestación urbana (1950-1969) se manifestó dando lugar al surgimiento de los actuales municipios de Puerto Rico, Capioví y Ruiz de Montoya. Esta etapa se caracterizó por consolidar los pueblos delineados durante la colonización.

Tras el trazado de las líneas en el mapa — cartografía — más la recuperación de la trama social —etnografía — se comenzó a vislumbrar la diversidad de las bases poblacionales de cada colonia. En este proceso, simultáneamente coexistieron compradores de lotes transoceánicos, descendientes de inmigrantes europeos, inmigrantes limítrofes y criollos que dejaron su huella tras la compra de un lote; mientras que la población aborigen quedó difuminada territorialmente, evidenciando la manera en quela mercantilización de la tierra otorgó derechos a los compradores, pero recluyó a la población originaria en los fundos fiscales o que no entraron bajo el régimen fundiario. Así, el espacio apropiado por los que habitan y transitan a Misiones y sus fronteras comenzó a ser representado cartográficamente para dar cuenta de una etnografía histórica que muestra una compleja formación de la alteridad regional y local.

\section{NOTAS}

${ }^{1} \mathrm{Al}$ considerar la documentación histórica de Misiones, se deben diferenciar dos períodos: uno anterior a la federalización (1881), etapa de la cual no se conservan documentos en archivos públicos de la provincia, pero sí fuera de ella. Debido a la di- 
áspora posjesuítica, los conflictos y movimientos de población que le sucedieron y la inestabilidad institucional quienes estudien este período deberán buscar información en los siguientes repositorios: Archivo General de la Nación,en Buenos Aires; Archivo Nacional de Asunción; Archivo General de Indias, en Sevilla; Biblioteca Nacional de Río de Janeiro; Archivo General de la Nación, en Montevideo; Archivo General de la provincia de Corrientes, entre otros. El siguiente período, posterior a la federalización, cuenta con algún grado de conservación y sistematización de documentación, particularmente la producida por las instituciones estatales, en la que la temática migratoria es prácticamente inexistente.

${ }^{2}$ La Compañía Eldorado realizó los siguientes folletos propagandísticos: Die Eldorado-KolonienimUrteilihrerBesucher [Las colonias de Eldorado a través del juicio de sus visitantes],1926; Die Eldorado Kolonien am Paraná-Fluss in Misiones-Argentinien [Las colonias de Eldorado sobre el Río Paraná en Misiones-Argentina], 1927; Das kolonisatorischeWerk der Eldorado-Gesellschaft [La obra colonizadora de la Compañía Eldorado], 1928; Die Entwicklung der Eldorado-Kolonien (1919-1929) [El desarrollo de las colonias de Eldorado (1919-1929)], 1929; LebenundTreiben in Eldorado [Vida y accionar en Eldorado], 1931; FortschrittundBlühen von Eldorado [Avance y florecimiento de Eldorado], 1934; Eldorado-Victoria, Monte Carlo, Puerto Rico-San Alberto, 1939. El único folleto de la «Compañía Colonizadora Alto Paraná» del año 1922 o 1923 también estaba redactado en alemán.

${ }^{3}$ En algunos casos, al no constarla fecha de venta, se utilizó como referencia la fecha de escrituración.

${ }^{4}$ En el momento de volcar la información a la base de datos, se cargó la totalidad de la información existente en los ficheros de la compañía, por lo tanto en algunos casos la información disponible llega hasta el presente. Paralos fines analíticos se tomaron únicamente las unidades que abarcaron el período 1919-1969.

${ }^{5}$ Para realizar este paso, se consultó bibliografía específica o se revisaron árboles genealógicos de familias alemanas-brasileñas (FOUQUET y SALVADOR de MOYA, 1962 TI y TII, 1965 TIII y T IV, HERKENHOFF et. al., 1975 y 1989; HUNSCHE, 1975; PORTO, 1996; y SCHWAB, 1997), en otros casos, corroboró el lugar de nacimiento a través de la base de datos realizada sobre las actas matrimoniales. Asimismo, ante la dificultad de establecer con precisión el origen étnico de algunos apellidos, se realizaron consultas a informantes clave con las dudas pertinentes, entre los que se pueden citar a Elida Arenhard, Marcelo Marek, Karina Dohmann, Margarita Kapler de Nedel y Juan Rodolfo Würgler.

${ }^{6}$ Agradezco a mis hermanos Ignacio y José por encargarse del diseño y de la elaboración de los mapas correspondientes al proceso de ocupación de Puerto Rico.

${ }^{7}$ Agradezco a Juan Plocherpor facilitarme las listas con los apellidos ordenados alfabéticamente de los propietarios y su correspondiente identificación de origen, elaboradasrespectivamente por Emilio Erhardt (23-11-1998) y Heiner Müller (19-11-1998).Erhardt nació el 9 de agosto de 1913; y Müller, el 6 de abril de 1910, ambos en Alemania.

${ }^{8}$ Juan Plocher aclara que en orden numérico le siguen: «8 de argentinos, 5 de suizos, 3 de africanos [alemanes-africanos] y 1 de un norteamericano» (PLOCHER, 2002).

${ }^{9}$ Charla mantenida con Juan Plocher, 16 -08- 2006.

${ }^{10}$ Agradezco a Fernando Jesús Jiménez por su meticulosa labor de búsqueda e identificación de la nacionalidad de los inmigrantes en esta base, así como por la comparación de datos en Family.search. También agradezco a Mónica López, quien trabaja en la biblioteca del CEMLA, por ayudar a identificar a los inmigrantes en la base de datos con apellidos de tres letras, tales como Ehe, Dam, Hak, etc., los cuales no figuran en línea.

\section{BIBLIOGRAFÍA Y FUENTES CONSULTADAS}

\section{Fuentes cartográficas}

1.1 Dirección General de Catastro de la Provincia de Misiones, Posadas.

- Plano de la Colonia Montecarlo: del Río Caraguatay al Itacuruzú. Escala $1: 25.000$. [s.f.]

- Departamento Libertador General San Martín, 110 x 115 cm. Escala 1:50000. 1974. 
- Municipio Puerto Rico, 76 X $80 \mathrm{~cm}$. Escala 1:25000. 1967

- Municipio Ruiz de Montoya, 80 x $60 \mathrm{~cm}$. Escala 1:25000. [s.f.]

- Municipio Capioví, $46 \times 74 \mathrm{~cm}$. Escala 1:25000. [s.f.]

- Municipio Leoni, 63 x 53 cm. Escala 1:25000. [s.f.]

- Mapa catastral, político y vial, unificación de las hojas de cartas topográficas, Escala 1:250000.1981.

- Pueblo Libertador General San Martín [Puerto Rico], Compañía Eldorado Ltda. S.A., Escala 1:50000. [s.f.]

- Colonia Eldorado -9 de Julio. Compañía Eldorado Ltda. S.A., Escala 1:30000. [s.f.].

\subsection{Mapas y planos antiguos en repositorios particulares}

- Misiones, elaboradopor Francisco Foulliand, 1909.

- Colonias Puerto Rico y San Alberto, Compañía Colonizadora Alto Paraná. Escala 1:25000. c.1920.

- Misiones, Patrón de Asentamiento y su Evolución Histórica, Secretaría de Planeamiento, realizado por Miguel AngelStefañuk, Escala 1:500000. Actualización 2000.

- Colonia Eldorado, 1927, 1930, 1939, 1945.

- Colonia Montecarlo,Compañía Colonizadora Alto Paraná. Escala 1:25000. c. 1920 .

- San Alberto, Escala 1:11500. [s.d.]

- San Alberto, Escala 1:5000. [s.d.]

- Alto Paraná, Red Caminera y Colonias. Cartógrafo H. F. Hassel. Escala 1:750000. [s.f.]

\section{BIBLIOGRAFÍA GENERAL}

AA.VV. Jornadas sobre Poblamiento, Colonización e Inmigración en Misiones. Posadas: Ed. Montoya, 1999, 2001, 2003, 2005, 2007, 2009.

ABINZANO, R. (1985). Procesos de integración en una sociedad multiétnica. La provincia argentina de Misiones (1880-1985). Tesis Doctoral, Universidad de Sevilla.

ARENHARDT, Elida (2003) "Don Adolfo Schwelm y su proyecto colonizador", en: III Jornadas sobre Poblamiento, Colonización e Inmigración de Misiones.Posadas, Ed. Montoya, p. 156-166.

BARANGER, D. (1999) Construcción y análisis de datos.Posadas: Ed. Universitaria de Misiones.

BARTOLOMÉ, L. (1982). Colonias y Colonizadores en Misiones, Posadas, Instituto de Investigación Facultad de Humanidades y Ciencias Sociales, UNaM. BARTOLOMÉ, L. (2000). Los Colonos de Apóstoles.Posadas, Editorial Universitaria.

BELASTEGUI, H. (2004). Los colonos de Misiones. Posadas: Ed. Universitaria de Misiones.

036 BLOCH, M. (2002).La tierra y el campesino. Agricultura y vida rural en los siglos XVII y XVIII. Barcelona, Crítica.

BLOCH, M. (2006). Les caractères originaux de l'historie rural française. Paris, Agora.

BOLSI, A. (1986). "Misiones (una aproximación geográfica al problema de la yerba mate y sus efectos en la ocupación del espacio y el poblamiento)"; en: 
Folia Histórica del Nordeste, Resistencia, IIGHI-CONICET-FUNDANORD, (7), 9-253.

BRAUDEL, F. (2001). El Mediterráneo y el mundo mediterráneo en la época de Felipe II. Madrid, FCE.

BRIONES, C. (2005). Cartografia Argentinas. Políticas indigenistas y formaciones provinciales de alteridad. Buenos Aires: Antropofagia.

CARBONELL de MASY, R. (1985). Grupos étnicos y cooperativas agrícolas en Misiones, Posadas, Departamento de Cooperativismo de la Facultad de Ciencias Económicas de la UNaM.

CARDOSO de OLIVEIRA, R. (1992). Etnicidad y Estructura Social.México, Ciesas.

CARRERA DIEZ, G. (2007). La cartografía etnográfica como herramienta técnica y metodológica en investigaciones antropológicas: el caso de Constantina. Cuadernos de Los Amigos de Los Museos de Osuna, (9), 72-81.

CEBOLlA BADIE, M. (2016). Naturaleza y Cosmología Mbya. Buenos Aires,Biblos.

DE MARCO, G. (1980). "El Nordeste Misionero: su poblamiento entre los años 1895 y 1970, Contribución a la geografía histórica de la Argentina". En: I Encuentro de Geohistoria Regional, Resistencia, IIGHI-CONICET, 1980: 213-261.

EIDT, R. (1971). Pioneer settlement in Northeast Argentina, Madison, The University of Wisconsin Press.

FOGELER, M. R. (2007). Etnografia y red de parentesco de los colonos escandinavos en las Sierras Centrales de Misiones. Tesis de Maestría, Universidad Nacional de Misiones.

FOUQUET, Carlos y SALVADOR DE MOYA, Coronel [dir.] (1962) Subsídios Genealógicos: Famílias Brasileiras de origemGermânica, Tomo I y II. São Paulo. Instituto Genealógico Brasileiro e Instituto Hans Staden.

FOUQUET, Carlos y SALVADOR DE MOYA, Coronel [dir.] (1965) Subsídios Genealógicos: Famílias Brasileiras de origemGermânica, Tomo III y IV. São Paulo. Instituto Genealógico Brasileiro e Instituto Hans Staden.

FOUQUET, Carlos y SALVADOR DE MOYA, Coronel [dir.] (1967) Subsídios Genealógicos: Famílias Brasileiras de origemGermânica, Tomo V. São Paulo. Instituto Genealógico Brasileiro e Instituto Hans Staden.

GALLERO, C. (2008). El llamado del Oro Verde. Memorias de inmigrantes suizos en Misiones. Buenos Aires, Araucaria Editora.

GALLERO, C. (2009 a). Con la patria a cuestas. La inmigración alemana-brasileña en Misiones. Buenos Aires, Araucaria Editora, Instituto de Investigaciones Geohistóricas.

GALLERO, C. (2009 b). "La inmigración suiza en Misiones, Argentina". En:LArevista - el boletín de la Sociedad Suiza de Americanistas (SSA). Número 71, 33-43.

GALLERO, C. (2010). "La territorialización de la germaneidad en los alemanes-brasileños de Misiones, Argentina”, en: Iberoamericana. América Latina - España - Portugal,Año IX, N. . 39, pp. 77-104 .

GALLERO, C. (2012). "La inmigración alemana-brasileña en Misiones, Argentina", en: Estudios Migratorios Latinoamericanos, Año 26, N. ${ }^{\circ} 73$, JulioDiciembre 2012, 29-49.

GALLERO, C. (2016)."Las Particularidades de la inmigración brasileña en la Argentina". Cadernos ObMigra-Revista Migrações internacionais, vol. 2, (1), 125-155. 
GALLERO, C. (2017). “Colonización y Ocupación no planificada. La mercantilización de la tierra agrícola en Misiones (1920-2000)". En: Travesía. Revista de historia económica y social. Vol. 19, (1) 77-109.

GALLERO, C. y CEBOLLA BADIE (2011). "Las relaciones blanco-indio a través del registro fotográfico en Misiones, Argentina (1920-1960)" .En: GIORDANO, M. y REYERO, A. (Comp.), Identidades en foco. Fotografia e investigación social, Resistencia, IIGHI-UNNE.

GALLERO, C. y CEBOLLA BADIE (2016). "Eran sólo indios... la construcción de la alteridad mbya-guaraní en el Alto Paraná de Misiones, Argentina (1920-1960)". En: Cadernos do Lepparq, Vol. XIII, № 26, ISSN 2316-8412.

GALLERO, C. y KRAUSTOFL, E. (2009). "Proceso de poblamiento y migraciones en la Provincia de Misiones, Argentina (1881-1970)". En: Revista de Antropología Avá (16), 245-264.

GARAVAGLIA, J. C., y GAUTREAU, P. Eds. (2011). Mensurar la tierra, controlar el territorio. America Latina, siglos XVIII- XIX. Rosario: Prohistoria.

GOROSITO KRAMER, A. (1982). Encuentros y Desencuentros. Relaciones inter-étnicas y representaciones en Misiones, Argentina. Tesis de Maestría, Universidad de Brasilia.

GUBER, R. (2004). El salvaje metropolitano. Reconstrucción del conocimiento social en el trabajo de campo. Buenos Aires, Editorial Paidós.

GUBER, R. (Comp.) (2014). Prácticas Etnográficas. Buenos Aires: Miño y Dávila.

GUBER, R. (Coord. Gral.) (2018) Trabajo de campo en América Latina. Buenos Aires: SB.

HARLEY, J. B. (2005): La nueva naturaleza de los mapas. Ensayos sobre la historia de la cartografía. México: FCE.

HERKENHOFF, E. y BÖBEL, M. (1989) Famílias Brasileiras de origemGermânica, Tomo VII. São Paulo: Instituto Genealógico Brasileiro e Instituto Hans Staden.

HERKENHOFF, E. y HERKENHOFF, R. (1975) Famílias Brasileiras de origemGermânica, Tomo VI. São Paulo: Instituto Genealógico Brasileiro e Instituto Hans Staden.

HEUSER, E. [c. 1980]: Memorias sobre Misiones. Inédito.

HUNSCHE, C. (1975) O Biênio 1824/25 da imigração e colonizaçãoalemã no Rio Grande do Sul: província de São Pedro, Porto Alegre, A Nação, 2. ed.

KEGLER, R. (2006). Los alemanes en Misiones. Posadas: Edición del Autor.

KOPP, Thomas (1949) Die SiedlungimWalde, Buenos Aires, Verlag "El buen libro".

KRAUSTOFL, E. (2007). Colonización, Inmigración y Etnicidad en la Provincia de Misiones, Argentina. La colonización privada de inmigrantes polacos en las colonias de Wanda y Gob. J.J.Lanusse. Informe Final del Proyecto. Secretaría de Investigación y Posgrado, FHyCS-UNaM.

LOIS, C. (2014). Mapas para la nación: episodios en la historia de la cartografia argentina. Buenos Aires,Biblos.

MEDING, H. (1995). "Etnicidad, identidades y migraciones de los colonos de habla alemana en Misiones". En: Estudios Migratorios Latinoamericanos (31), 727-745.

MENENDEZ, E. (1971). "Informe sobre las migraciones brasileñas en Misiones". En: Estudios sobre el nivel de vida de la población rural de Misiones. Posadas: Dirección General de Estadística de la Provincia de Misiones. Consultores Técnicos SA. 
MICOLIS, M. (1973). Une CommunautéAllemande en Argentina: Eldorado (Problèmes d intégration socio-culturelle).Quebec: Centre International de reserches sur le bilinguisme.

MONTOYA ARANGO, V. (2007). "El mapa de lo invisible. Silencios y gramática del poder en la cartografía". En: Universitas humanística. Número 63, pp. 155-179.

NIELLA, G. (2002). Los trabajos de Don Juan. El Paraguay a través de una historia de Vida. Tesis de Grado, Universidad Nacional de Misiones.

PERIÉ de SCHIAVONI, A. y ZOUVI, S. (1985). La Colonización en Misiones: $1^{\circ}$ Parte (Contexto internacional, nacional y sus antecedentes), Posadas, Centro de investigaciones histórico-Culturales, Instituto de Investigación, Facultad de Humanidades y ciencias sociales, UNaM.

PICCOLOTTO SIQUEIRA BUENO, B. (2004) "Decifrando mapas: sobre o conceito de "território" e suas vinculações com a cartografia". En: Anais do Museu Paulista. São Paulo. V. 12, pp. 193-234.

PLOCHER, J. (2002). "Las nacionalidades de los propietarios de lotes de Montecarlo adquiridos entre 1920 y 1943". En: IV y V Seminário Nacional de Pesquisadores da História das Comunidades Teuto-brasileiras, Lajeado, 2002, pp. 213-214.

PORTO, A. (1996), O trabalhoAlemão no Rio Grande Do Sul, Porto Alegre, MartinsLivreiro Editor.

RAUBER, G. y KUHN, L. (2012 y 2014). Alemanes de Alemania en los inicios de la colonización de Puerto Rico. Puerto Rico: Ediciones Germania.

SANTOS, M. (1996). Metamorfosis del espacio habitado. Barcelona: Oikos Tau.

SAUER, C. (1976) El factor temporal. En: Patricio Randle (Ed.), Teoría de la Geografia, Primera Parte. Buenos Aires: GAEA Sociedad Argentina de Estudios Geográficos, Serie Especial (3), 237-271.

SCHIAVONI, G. (1998). Colonos y ocupantes. Parentesco, reciprocidad y diferenciación social en la frontera agraria de Misiones. Posadas: Editorial Universitaria.

SCHWAB, C. (1997) Resumo Histórico e Genealogía dos Alemães do Volga, Ponta Grossa, Schwab.

SELLIER, J. (2008) Atlas de los Pueblos de Europa Occidental, Barcelona, Paidós.

SEYFERTH, G. (2000). As identidades dos imigrantes e o MeltingPot Nacional. En: Horizontes Antropológicos, Porto Alegre,UFRGS. IFCH, Programa de PosgradoenAntropología Social, año 6 (14), 143-176.

SNIHUR, E. A. (1997) De Ucrania a Misiones. Una experiencia de Transformación y crecimiento, Apóstoles: Colectividad Ucraniana de Misiones.

STEFAÑUK, M. A. (1995). Evolución dela cartografía de Misiones. Posadas: Ediciones Montoya.

STEFAÑUK, M. A. (1999). Patrón de Asentamiento: su evolución histórica en la Provincia de Misiones. In: I Jornadas de Poblamiento, Colonización e Inmigración en Misiones. Posadas: Ediciones Montoya, pp. 301-310.

STEFAÑUK, M. A. (2009). Diccionario Geográfico Toponímico de Misiones. Buenos Aires, Contratiempo Ediciones.

YAMAGUCHI, M. L. (2000). “Uds. Los Gaijin”. La inmigración japonesa en Colonia Lujan, Misiones, Argentina. Tesis de Grado, Universidad Nacional de Misiones. 